\title{
CARACTERÍSTICAS DEFINIDORAS E FATORES ASSOCIADOS À OCORRÊNCIA DAS SÍNDROMES HIPERTENSIVAS GESTACIONAIS*
}

\author{
Taize Sbardelotto ${ }^{1}$ Érica de Brito Pitilin² ${ }^{2}$ Janine Schirmer ${ }^{3}$, Maicon Henrique Lentsck ${ }^{4}$, Débora \\ Tavares de Resende e Silva ${ }^{5}$, Larissa Hermes Thomas Tombini ${ }^{6}$
}

\begin{abstract}
RESUMO: Objetivo: identificar as características definidoras e os fatores relacionados em Síndromes Hipertensivas Gestacionais. Método: estudo longitudinal, retrospectivo, realizado no centro de saúde de pré-natal de alto risco, num município do oeste catarinense em 2016. Como critérios de inclusão adotou-se: gestação única, que não tenha resultado em aborto ou óbito fetal, a data da última menstruação entre janeiro a dezembro de 2015, e a presença de diagnóstico da síndrome. Para a análise dos dados foi utilizado o software SPSS, versão 20.0. Resultados: foram analisados 90 prontuários, sendo reveladas as características definidoras dos diagnósticos pela elevação da pressão arterial e a presença de proteinúria. Os fatores relacionados foram: idade materna, ganho de peso, índice de massa corporal, antecedentes de doenças hipertensivas, número de consultas pré-natal e o uso de medicação anti-hipertensiva. Conclusão: identificou quais características predizem com maior exatidão a ocorrência do diagnóstico investigado, e a redução das formas graves da doença.
\end{abstract}

DESCRITORES: Saúde da mulher; Hipertensão induzida pela gravidez; Perfil de saúde; Cuidado pré-natal.

\section{DEFINING CHARACTERISTICS AND FACTORS ASSOCIATED WITH THE OCCURRENCE OF GESTATIONAL HYPERTENSIVE SYNDROMES}

\begin{abstract}
Objective: to identify the defining characteristics and related factors of Gestational Hypertensive Syndromes Method: longitudinal retrospective study performed at a high-risk prenatal health center in a city in the west of Santa Catarina in 2016. The inclusion criteria were singleton pregnancy that did not result in miscarriage of intrauterine fetal death, date of last menstrual period between January and December 2015 and diagnosis of Gestational Hypertensive Syndrome. SPSS software, version 20.0 was used in data analysis. Results: 90 medical records were analyzed, and the defining characteristics of the diagnoses were revealed by high blood pressure and the presence of proteinuria. The related factors were maternal age, weight gain, body mass index, history of hypertensive diseases, number of prenatal consultations and use of antihypertensive medication. Conclusion: the characteristics that more accurately predict the occurrence of the diagnosis investigated and the reduction of the severe forms of the disease were identified.
\end{abstract}

KEYWORDS: Women's health; Pregnancy-induced hypertension; Health profile; Prenatal care.

\section{CARACTERÍSTICAS DEFINIDORAS Y FACTORES ASOCIADOS A LA OCURRENCIA DE LOS SÍNDROMES HIPERTENSIVOS GESTACIONALES}

RESUMEN: Objetivo: identificar las características definidoras y los factores relacionados en Síndromes Hipertensivos Gestacionales. Método: estudio longitudinal, retrospectivo, realizado en el centro de salud de prenatal de alto riesgo, en un municipio del oeste de Santa Catarina en 2016. Como criterios de inclusión se adoptaron: gestación única que no tenga resultado en aborto u óbito fetal, la fecha de la última menstruación entre enero y diciembre de 2015, y la presencia de diagnóstico del síndrome. Para el análisis de los datos se utilizó el software SPSS, versión 20.0. Resultados: se analizaron 90 prontuarios, siendo reveladas las características definidoras de los diagnósticos por la elevación de la presión arterial y la presencia de proteinuria. Los factores asociados fueron: edad materna, aumento de peso, índice de masa corporal, antecedentes de enfermedades hipertensivas, número de consultas prenatal y uso de medicación anti hipertensiva. Conclusión: se identificaron cuales características apuntan con más exactitud la ocurrencia del diagnóstico investigado, y la reducción de las formas graves de la enfermedad.

DESCRIPTORES: Salud de la mujer; Hipertensión inducida por la gravidez; Perfil de salud; Cuidado prenatal.

\footnotetext{
* Artigo extraído do Trabalho de Conclusão de Curso intitulado: Análise das Síndromes Hipertensivas Gestacionais em um Município do Oeste Catarinense. Universidade Federal da Fronteira Sul, 2016.

'Enfermeira. Graduada em Enfermagem pela Universidade Federal da Fronteira Sul Chapecó, SC, Brasil.

2Enfermeira. Doutoranda pela Universidade Federal de São Paulo. Professora Assistente do Departamento de Enfermagem da Universidade Federal Fronteira Sul. Chapecó, SC, Brasil..

${ }^{3}$ Enfermeira. Doutora em Enfermagem Materno-Infantil pela Universidade Federal de São Paulo. Professora titular do departamento de Enfermagem, diretora da escola Paulista de Enfermagem. São Paulo, SP, Brasil.

${ }^{4}$ Enfermeiro. Doutorando em Enfermagem pelo Programa de Pós-graduação em Enfermagem da Universidade Estadual de Maringá/ PR. Professor Assistente do Departamento de Enfermagem da Universidade Estadual do Centro-Oeste. Maringá, PR, Brasil.

${ }^{5}$ Fisioterapeuta. Pós-doutora em Imunologia pela Universidade de São Paulo. Professora Assistente do Departamento de Enfermagem da Universidade Federal Fronteira Sul. Chapecó, SC, Brasil.

${ }^{6}$ Enfermeira. Mestre em Enfermagem pela Universidade Federal de Santa Catarina. Professora Assistente do Departamento de Enfermagem da Universidade Federal Fronteira Sul. Chapecó, SC, Brasil.
} 


\section{INTRODUÇÃO}

As Síndromes Hipertensivas Gestacionais (SHG) são consideradas umas das mais importantes complicações durante o ciclo gravídico. A incidência varia entre 6\% a 30\% das gestações, contribuindo para elevados índices de morbidade materna, e a principal causa de morte no mundo ${ }^{(1)}$.

As complicações dos distúrbios hipertensivos como pré-eclâmpsia e eclâmpsia correspondem a $25 \%$ de todas as mortes maternas em todo mundo, e, as principais causas de nascimentos prematuros, enquanto que no Brasil, esses números correspondem a $20 \%{ }^{(2-3)}$.

As SHG são definidas pelo National High Blood Pressure Education Program (NHBPEP) - Programa Nacional de Educação de Pressão Arterial - como Hipertensão Gestacional (HG), Hipertensão Arterial Crônica (HAC), Pré-eclâmpsia (PE) e Hipertensão Arterial Crônica sobreposta à Pré-eclâmpsia (HAC+PE) (4).

Entre as complicações maternas destacam-se trombocitopenia, aumento das enzimas hepáticas, hemólise das hemácias, Síndrome Hellp - Hemolytic anemia, Elevated Liverenzymes, Low Plateletcount) e Eclâmpsia; enquanto que no feto ocorre o comprometimento de seu desenvolvimento, o parto prematuro, baixo peso ao nascer e morte perinatal ${ }^{(5-6)}$.

A identificação das pacientes de risco para o desenvolvimento das SHG, seja por fatores relacionados à sua ocorrência, ou por características definidoras de suas modalidades, podem contribuir para uma redução da imprecisão na escolha diagnóstica para as situações/problemas apresentadas, prevenindo ou retardando a apresentação clínica das formas mais graves ${ }^{(7)}$.

As características definidoras são um conjunto de evidências que se agrupam como manifestação dos sinais e sintomas que asseguram a presença de um determinado diagnóstico, enquanto que os fatores relacionados, são aqueles que se constituem como a etiologia desse diagnóstico, ou seja, determinam as causas da origem de um determinado fenômeno(8)

A partir destas medidas é possível identificar quais características, dentre o conjunto de características, predizem com maior exatidão a ocorrência do diagnóstico investigado, dentro do contexto das SHG. Estudos que visem a melhor definição e aplicação prática destes diagnósticos podem ajudar a tornar o processo de inferência diagnóstica mais fidedigna ${ }^{(5)}$.

Atualmente não existe nenhum teste para a predição da SHG largamente aceito, ressaltando a importância de medidas preventivas que poderiam prolongar a gestação e diminuir os riscos maternos e fetais ${ }^{(3)}$. Diante disso, percebe-se que ainda são incipientes os estudos que abordam os fatores relacionados ao desenvolvimento das $\mathrm{SHG}$, devendo ser cada vez mais valorizados ${ }^{(5)}$.

Este estudo parte do pressuposto de que as gestantes hipertensas melhores assistidas, resultarão em menor chance de complicações advindas com o advento da doença instalada. Assim, objetivou-se identificar as características definidoras das SHG e os fatores que podem influenciar ou não a sua ocorrência.

\section{METODOLOGIA}

Trata-se de um estudo longitudinal, retrospectivo, realizado a partir da análise dos prontuários eletrônicos das gestantes com SHG encaminhadas ao serviço de referência para o acompanhamento do pré-natal de alto risco no município de Chapecó, Santa Catarina (SC), referência para os serviços de alta complexidade da região ${ }^{(9)}$.

Os prontuários foram selecionados segundo os critérios de inclusão: gestação única, que não resultaram em aborto ou óbito fetal, data da última menstruação (DUM) de 2015 e diagnóstico de SHG. Foram excluídos os prontuários que não apresentavam a confirmação diagnóstica. A amostra resultou em 90 prontuários. 
Os dados foram coletados a partir do acesso ao programa eletrônico do serviço de saúde, por meio de um instrumento semiestruturado com informações referentes às características sociodemográficas, condições de saúde, antecedentes pessoais e familiares de doenças hipertensivas, medicação, aspectos clínicos laboratoriais (idade gestacional - IG), níveis pressóricos (considerada normal PA $<140 / 90$ $\mathrm{mmHg}$ ), avaliação nutricional (baixo peso, adequada, sobrepeso, obesidade), índice de massa corpórea (IMC), aferição da altura uterina (AU) e, ausculta do batimento cardíaco fetal (BCF).

Ainda, foram analisados: o número de consultas pré-natal, proteinúria (presente, a partir do exame de fita Labstixe considerando 2+ como o diagnóstico provável), hematócrito, hemoglobina; desfecho neonatal (tipo de parto, idade do nascimento - termo precoce: 37 semanas a 38 semanas e seis dias; termo completo: 39 semanas a 40 semanas e seis dias; termo tardio: 41 semanas a 41 semanas e seis dias; pós-termo: a partir de 42 semanas); peso do nascimento e Apgar no $1^{\circ}$ e $5^{\circ}$ minuto de vida (sem asfixia: 8 a 10, asfixia leve: 7 a 5, asfixia moderada 4 e 3, asfixia grave 2 a 0).

As variáveis dependentes foram aquelas relacionadas ao desfecho das SHG classificadas em: HG, aumento da pressão arterial após a $20^{a}$ semana de gestação, sem presença de proteinúria, normatizando seus níveis pressóricos até a $12^{a}$ semana do puerpério; HAC, definida como a elevação da pressão identificada antes da gestação ou até a 20 $0^{a}$ semana, permanecendo elevada após as 12 semanas do puerpério; PE a qual surge após a 20 ${ }^{a}$ semana, acompanhada de proteinúria em nível igual ou maior de $0,3 \mathrm{~g}$ em período de até 24 horas e HAC+PE que consiste no surgimento da PE em mulheres que já desenvolveram a HAC ou doença renal, gerando, concomitantemente, após a 20ª semana a proteinúria ${ }^{(4)}$.

A classificação da SHG foi definida, unicamente, ao momento em que foi diagnosticada durante a gestação. As variáveis independentes foram aquelas observadas a partir da primeira consulta do trimestre gestacional verificadas em três momentos - folow up (1으, $2^{\circ}$ e $3^{\circ}$ trimestre).

No que tange à confidencialidade dos dados assegurou-se o anonimato por um código caracterizado pela inicial " $\mathrm{P}$ ", da palavra "prontuário", acompanhado do algarismo arábico referente à ordem da coleta (P1, P2, P3...).

Para a análise dos dados foi utilizado o software SPSS, versão 20.0. Utilizou-se a estatística descritiva e análise bivariada por meio do teste Qui-quadrado de Pearson e, para a avaliação das afirmações das médias dos trimestres foi realizado o teste ANOVA. Todos os testes estatísticos inferenciais tiveram nível de significância $p<0,05$ e a qualidade do ajuste avaliada pelo teste de Hosmer-Lemeshow.

O referencial teórico utilizado para interpretação dos dados foi proposto pelas recomendações internacionais American Congress Of Obstetrician and Gynecogistis Executive Sumary ${ }^{(10)}$.

O desenvolvimento desta pesquisa seguiu as normas e diretrizes éticas, sendo aprovada pelo Comitê de Ética em Pesquisa com Seres Humanos (CEP) da Universidade Federal da Fronteira Sul (UFFS), sob o parecer n. $\stackrel{0}{1} 1.621 .337$.

\section{RESUltados}

As características das gestantes com SHG segundo condições sociodemográficas, história reprodutiva, fatores de risco para doenças crônicas hipertensivas e desfecho neonatal encontram-se na Tabela 1. 
Tabela 1 - Características das gestantes com SHG segundo condições sociodemográficas, história reprodutiva, fatores de risco para doenças hipertensivas e desfecho neonatal. Chapecó, SC, Brasil, 2016. (continua)

\begin{tabular}{|c|c|c|c|}
\hline \multirow{2}{*}{\multicolumn{2}{|c|}{ Variáveis }} & \multicolumn{2}{|c|}{$\mathrm{n}=\mathbf{9 0}$ gestantes } \\
\hline & & Frequência & $\%$ \\
\hline \multicolumn{4}{|l|}{ Idade } \\
\hline & $15-19$ & 01 & 1,1 \\
\hline & $20-39$ & 81 & 90 \\
\hline & $\geq 40$ & 08 & 8,9 \\
\hline \multicolumn{4}{|l|}{ Escolaridade } \\
\hline \multirow[t]{4}{*}{ (anos de estudo) } & $1-4$ & 42 & 46,7 \\
\hline & $5-8$ & 36 & 40 \\
\hline & $9-12$ & 04 & 4,4 \\
\hline & $\geq 12$ & 08 & 8,9 \\
\hline \multicolumn{4}{|l|}{ Estado Civil } \\
\hline & Solteira & 08 & 8,8 \\
\hline & Casada & 53 & 58,8 \\
\hline & Separada & 01 & 1,1 \\
\hline & Ignorado & 28 & 31,1 \\
\hline \multicolumn{4}{|l|}{ Cor } \\
\hline & Branca & 72 & 80 \\
\hline & Parda & 12 & 13,3 \\
\hline & Ignorado & 06 & 6,6 \\
\hline \multicolumn{4}{|c|}{ Situação Ocupacional } \\
\hline & Desempregada & 15 & 16,6 \\
\hline & Empregada & 27 & 30 \\
\hline & Ignorado & 48 & 53,3 \\
\hline \multicolumn{4}{|c|}{ História Reprodutiva } \\
\hline & Nulípara & 22 & 24,4 \\
\hline & Multípara & 68 & 76,6 \\
\hline & Aborto & 22 & 24,4 \\
\hline & Natimorto & 04 & 4,4 \\
\hline & Baixo peso ao nascer & 08 & 14,3 \\
\hline \multicolumn{4}{|l|}{ Tabagismo } \\
\hline & Sim & 05 & 5,5 \\
\hline & Não & 85 & 94,4 \\
\hline \multicolumn{4}{|l|}{ Tipo de parto } \\
\hline & Vaginal & 31 & 34,4 \\
\hline & Cirúrgico & 59 & 65,6 \\
\hline \multicolumn{4}{|c|}{ Idade Gestacional Nascimento } \\
\hline & Pré-termo & 14 & 15,6 \\
\hline & Termo precoce & 24 & 26,7 \\
\hline & Termo completo & 28 & 31,1 \\
\hline & Termo tardio & 24 & 26,7 \\
\hline
\end{tabular}




\begin{tabular}{lccc}
\hline Escore Apgar & & \\
& Sem asfixia $(8-10)$ & 28 & 31,1 \\
& $\begin{array}{c}\text { Asfixia leve }(7-5) \\
\text { Ignorado }\end{array}$ & 06 & 6,6 \\
& & 56 & 62,2 \\
\hline Peso ao nascer & $\leq 2,500 \mathrm{~g}$ & 08 & 8,8 \\
& $>2,500 \mathrm{~g}$ & 48 & 53,3 \\
& Ignorado & 34 & 37,7 \\
\hline História prévia doenças hipertensivas pessoais & & 42 & 46,7 \\
\hline História prévia doenças hipertensivas familiares & & 12 & 13,3 \\
\hline Uso anti-hipertensivo oral & 32 & 35,6 \\
\hline
\end{tabular}

A média de idade foi de 30,3 anos ( $\pm 5,9$ anos), com destaque para a faixa etária entre 20 a 39 anos, baixa escolaridade, casadas, brancas e multíparas. Em relação ao desfecho neonatal, merecem destaque os partos cirúrgicos, no termo completo, sem asfixia ao nascimento e peso maior que 2,500 g.

A Tabela 2 revela a ocorrência de associação entre as formas clínicas da SHG e os fatores de risco relacionados ao seu diagnóstico, de acordo com o trimestre gestacional. A HAC foi mais comum no primeiro trimestre $32(35,6 \%)$, enquanto que a HG prevaleceu como diagnóstico principal no $2^{\underline{0}}$ trimestre, com significância estatística para a elevação da pressão arterial em níveis $\geq 140 / 90 \mathrm{mmHg}$. A PE mostrou associação no $3^{\circ}$ trimestre bem como proteinúria e obesidade.

Tabela 2 - Associação entre as formas clínicas de SHG e as variáveis estudadas segundo o trimestre gestacional. Chapecó, SC, Brasil, 2016

\begin{tabular}{|c|c|c|c|c|c|c|c|}
\hline \multirow[b]{3}{*}{ Síndrome Hipertensiva Gestacional (SHG) } & \multicolumn{7}{|c|}{ Trimestre } \\
\hline & \multicolumn{2}{|c|}{ Primeiro } & \multicolumn{2}{|c|}{ Segundo } & \multicolumn{2}{|c|}{ Terceiro } & \multirow[t]{2}{*}{ Valor-p } \\
\hline & $\mathbf{n}$ & $\%$ & $\mathbf{n}$ & $\%$ & $\mathbf{n}$ & $\%$ & \\
\hline Hipertensão Arterial Crônica (HAC) & 32 & $35,6^{*}$ & 29 & 32,2 & 13 & 14,4 & 0,023 \\
\hline Hipertensão Gestacional (HG) & - & - & 53 & 58,9 & 25 & 27,8 & \\
\hline Pré-eclâmpsia (PE) & - & - & 05 & 5,6 & 33 & $36,7^{*}$ & \\
\hline Hipertensão Arterial Crônica sobreposta PE (HAC+PE) & - & - & 03 & 3,3 & 19 & 21,1 & \\
\hline \multicolumn{8}{|l|}{ Aferição da Pressão Arterial } \\
\hline Valor normal $(<140 \times 90 \mathrm{mmHg})$ & 23 & 67,6 & 26 & 28,9 & 49 & 54,4 & $<0,001$ \\
\hline Valor alterado $(\geq 140 \times 90 \mathrm{mmHg})$ & 11 & 32,4 & 64 & $71,1^{*}$ & 41 & 45,6 & \\
\hline Proteinúria & & & & & & & $<0,001$ \\
\hline Negativa $(<2+$ fita reagente $)$ & 81 & 90 & 80 & 90 & 38 & 42,2 & \\
\hline Positiva ( $\geq 2+$ fita reagente) & 09 & 10 & 08 & 10 & 52 & $57,8^{*}$ & \\
\hline Estado Nutricional & & & & & & & 0,042 \\
\hline Baixo peso & 01 & 2,9 & 01 & 1,7 & 01 & 1,4 & \\
\hline Adequado & 01 & 2,9 & 03 & 5,2 & 07 & 9,5 & \\
\hline Sobrepeso & 11 & 32,4 & 11 & 19 & 18 & 24,3 & \\
\hline Obesidade & 21 & 61,8 & 43 & 64,9 & 48 & $74,1^{*}$ & \\
\hline \multicolumn{8}{|l|}{ Avaliação na consulta pré-natal } \\
\hline BCF & - & - & 33 & 36,7 & 68 & 75,6 & \\
\hline Altura uterina & - & - & 44 & 48,9 & 67 & 74,4 & \\
\hline
\end{tabular}

* Teste Exato de Fischer 
Ao comparar a evolução das variáveis peso, IMC, Pressão Arterial Sistólica (PAS) e Pressão Arterial Diastólica (PAD), segundo os trimestres gestacionais foi possível identificar significância estatística para o $3^{\mathrm{o}}$ trimestre, relacionado ao aumento do peso e ao IMC (Tabela 3).

Tabela 3 - Comparação das variáveis paramétricas de gestantes com SHG segundo o trimestre gestacional. Chapecó, SC, Brasil, 2016

\begin{tabular}{|c|c|c|c|c|c|c|c|}
\hline \multirow[b]{2}{*}{ Variáveis } & \multicolumn{2}{|c|}{ 10 Trimestre } & \multicolumn{2}{|c|}{ 20 Trimestre } & \multicolumn{2}{|c|}{$3^{\circ}$ Trimestre } & \multirow[b]{2}{*}{ P valor } \\
\hline & Média & $\pm \mathrm{SD}$ & Média & $\pm \mathrm{SD}$ & Média & $\pm \mathrm{SD}$ & \\
\hline Peso (Kg) & $88,00^{a}$ & 21,41 & $88,88^{\mathrm{a}}$ & 21,24 & $93,52^{b}$ & 19,73 & $<0,001$ \\
\hline IMC (Kg/Alt²) & $33,16^{a}$ & 7,43 & $33,48^{\mathrm{a}}$ & 7,33 & $35,20^{\mathrm{b}}$ & 6,73 & $<0,001$ \\
\hline PAS (mmHg) & 127,64 & 2,46 & 127,05 & 3,06 & 122,14 & 2,54 & 0,166 \\
\hline PAD (mmHg) & 81,76 & 16,23 & 81,44 & 11,84 & 81,44 & 15,54 & 0,995 \\
\hline
\end{tabular}

abTeste Post Hoc de Bonferroni: médias com diferenças significativas entre os grupos

A distribuição das características estudadas segundo as formas clínicas das SHG estão na Tabela 4. A PE esteve relacionada à idade entre 20 a 39 anos, níveis pressóricos $\geq 140 / 90 \mathrm{mmHg}$, proteinúria positiva $>2+$ e, mais de sete consultas no pré-natal. Por sua vez, a HAC+PE esteve relacionada com os antecedentes de doenças hipertensivas e ao uso de medicação anti-hipertensiva.

Tabela 4 - Distribuição das características sociodemográficas, clínicas, laboratoriais e neonatais, segundo as formas clínicas das SHG em gestantes no terceiro trimestre. Chapecó, SC, Brasil, 2016. (continua)

\begin{tabular}{|c|c|c|c|c|c|c|c|c|c|c|c|}
\hline \multirow[b]{2}{*}{ Variáveis } & \multirow[b]{2}{*}{$\mathbf{n}$} & Total & \multicolumn{2}{|r|}{$\mathrm{HC}$} & \multicolumn{2}{|c|}{ HG } & \multicolumn{2}{|c|}{$\mathrm{PE}$} & \multicolumn{2}{|c|}{$\mathrm{HC}+\mathrm{PE}$} & \multirow[t]{2}{*}{ P valor } \\
\hline & & $\%$ & $\mathbf{n}$ & $\%$ & $\mathbf{n}$ & $\%$ & $\mathbf{n}$ & $\%$ & $\mathrm{n}$ & $\%$ & \\
\hline Cor & & & & & & & & & & & $1,000 *$ \\
\hline Branca & 72 & 80 & 11 & 15,3 & 20 & 27,8 & 26 & 36,1 & 15 & 20,8 & \\
\hline Parda & 18 & 20 & 2 & 11,1 & 5 & 27,8 & 7 & 38,9 & 4 & 22,2 & \\
\hline Faixa etária & & & & & & & & & & & $0,034^{*}$ \\
\hline 15 a 19 anos & 1 & 1,1 & - & - & 1 & 100 & - & - & - & - & \\
\hline 20 a 39 anos & 81 & 90 & 9 & 11,1 & 23 & 28,4 & 32 & 39,5 & 17 & 21 & \\
\hline$>40$ anos & 8 & 8,9 & 4 & 50 & 1 & 12,5 & 1 & 12,5 & 2 & 25 & \\
\hline $\begin{array}{l}\text { Escolaridade (anos de } \\
\text { estudo) }\end{array}$ & & & & & & & & & & & $0,377^{*}$ \\
\hline 1 a 4 anos & 42 & 46,7 & 9 & 21,4 & 10 & 23,8 & 11 & 28,6 & 12 & 28,6 & \\
\hline 5 a 8 anos & 36 & 40 & 3 & 8,3 & 11 & 30,6 & 16 & 44,4 & 6 & 16,7 & \\
\hline 9 a 12 anos & 4 & 4,4 & 1 & 25 & 1 & 25 & 2 & 50 & - & - & \\
\hline$>12$ anos & 8 & 8,9 & - & - & 3 & 37,5 & 4 & 50 & 1 & 12,5 & \\
\hline Estado civil & & & & & & & & & & & $0,444^{*}$ \\
\hline Sem companheiro & 7 & 11,3 & 1 & 14,3 & 1 & 14,3 & 2 & 28,6 & 3 & 42,9 & \\
\hline Com companheiro & 55 & 88,7 & 7 & 12,7 & 16 & 29,1 & 23 & 41,8 & 9 & 16,4 & \\
\hline Ocupação & & & & & & & & & & & $0,305^{*}$ \\
\hline Desempregada & 15 & 35,7 & 3 & 20 & 3 & 20 & 5 & 33,3 & 4 & 26,7 & \\
\hline Empregada & 27 & 64,3 & 1 & 3,7 & 7 & 25,9 & 14 & 51,9 & 5 & 18,5 & \\
\hline Paridade & & & & & & & & & & & $0,289 *$ \\
\hline Mutípara & 68 & 75,6 & 12 & 17,6 & 18 & 26,5 & 26 & 38,2 & 12 & 17,6 & \\
\hline Primípara & 22 & 24,4 & 1 & 4,5 & 7 & 31,8 & 7 & 31,8 & 7 & 31,8 & \\
\hline
\end{tabular}




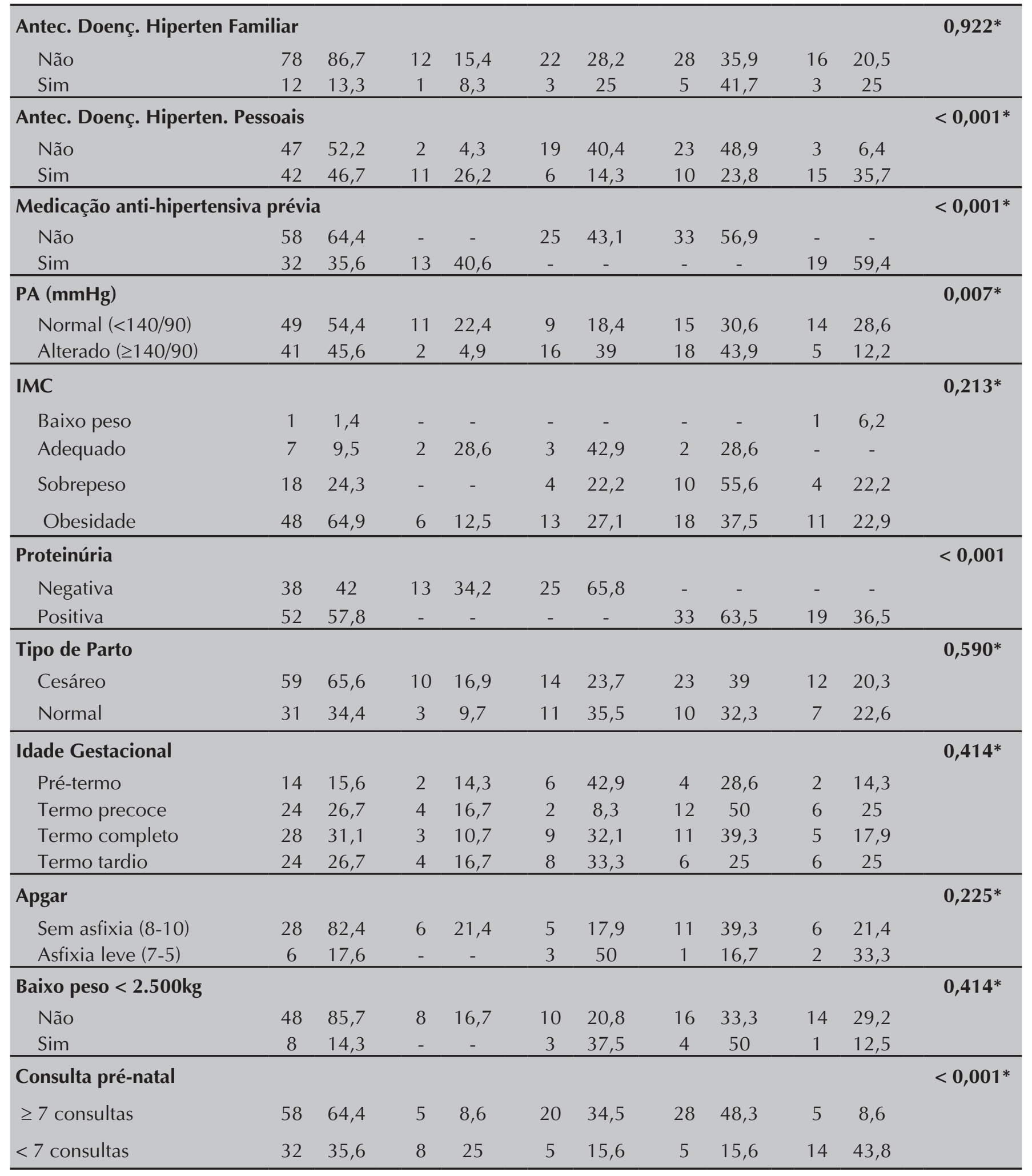

* Teste Exato de Fischer

\section{- DISCUSSÃO}

Entre os fatores relacionados à ocorrência das SHG identificados neste estudo, a idade materna mostrou relação à ocorrência da PE. As gestantes entre 20 a 39 anos foram as mais acometidas tendo em vista o período reprodutivo em que se encontram. Dados semelhantes também foram encontrados em outros estudos que apresentaram predominância da faixa etária nesse período ${ }^{(11-12)}$. 
Ainda que os extremos de idade representam potencial risco para as SHG, nesse estudo não houve significância estatística. Há controvérsia se a gravidez nos extremos no período procriativo eleva os riscos das síndromes hipertensivas ${ }^{(13)}$.

Quanto ao nível de escolaridade, poucos anos de estudo apresentaram maiores proporções, porém, não estando relacionada estatisticamente à ocorrência da síndrome. O grau de escolaridade de um indivíduo pode estar relacionado à sua capacidade de obter informação acerca dos cuidados sobre sua própria saúde, tornando-se vulnerável a desenvolver determinadas doenças.

Os achados corroboram com o argumento da NHBPEP que reforça que o nível socioeconômico não predispõe à população as diversas formas clínicas da $\mathrm{SHG}^{(4)}$. O contexto de cada gestação é determinante para o seu desenvolvimento, cabendo às equipes de saúde compreender os múltiplos fatores que podem ameaçar a saúde materna.

Demais fatores sociodemográficos também não mostraram associação com as formas clínicas da SHG. Tais fatores não configuraram risco para a ocorrência do desfecho, devido a amostra pertencer à classe menos favorecida e atendida em uma unidade pública de saúde ${ }^{(13)}$.Outra explicação pode ser dada pela diversidade racial, a qual é composta a sociedade brasileira, o que dificulta a estratificação de status raciais bem definidos.

Apesar da literatura indicar a prevalência de HG em primíparas, neste estudo o cenário em multíparas pareceu ser diferente, uma vez que a paridade não demonstrou associação significativa com as SHG. Um resultado semelhante foi encontrado à luz de outros estudos ${ }^{(12,14)}$. É possível que as gestantes deste estudo possuam características peculiares diferentes, como o início da idade em que começaram a engravidar.

No que se refere às formas clínicas da SHG, a HAC esteve relacionada ao seu surgimento no $1^{\circ}$ trimestre, reforçando a característica que define o seu diagnóstico, sendo um importante fator de risco para a sobreposição da PE. Na atual conjuntura social observa-se o aumento na probabilidade das mulheres serem portadoras de HAC durante a gravidez, ao postergarem a gestação para além dos 30 anos de idade, tornando-se necessárias as orientações pré-concepcional e assistência qualificada do pré-natal(15).

A utilização de anti-hipertensivos e antecedentes pessoais foram outros fatores relacionados à ocorrência da SHG com significância estatística para HAC+PE. Tais achados já eram esperados, uma vez que a pré-eclâmpsia foi o diagnóstico mais comum, e as doenças prévias associadas constituem fatores de risco para a ocorrência das síndromes hipertensivas. Gestantes que apresentem hipertensão na gravidez devem ser aconselhadas quanto ao risco de desenvolverem hipertensão em uma nova gestação.

Por sua vez, a ocorrência da HG mostrou associação a partir do $2^{\circ}$ trimestre com elevação dos níveis pressóricos conforme observado em outros estudos ${ }^{(5,8)}$. Os riscos advindos do aumento pressórico configuram-se em distúrbios de origem circulatória, além de fator de risco para sobrevida neonatal ${ }^{(16)}$.

A PE esteve relacionada ao $3^{\circ}$ trimestre, confirmada pelo aumento significativo da PA acompanhado da proteinúria, sendo diagnosticada pela presença de 2+ na fita reagente. A ausência de valores específicos do exame não impediu de verificar a associação com o desfecho, uma vez que não há relação entre a quantidade exata de proteína na urina, e, sim, sua ausência ou presença ${ }^{(17)}$.

O ganho de peso e a obesidade foram significativos no $3^{\circ}$ trimestre gestacional. $O$ aumento do peso no decorrer dos trimestres pode estar associado ao próprio crescimento fetal ou ao acometimento pela hipertensão gestacional. Outro estudo também corrobora com o aumento do ganho de peso em gestantes hipertensas, destacando o comprometimento do percurso gestacional em obesas ${ }^{(13)}$.

Em relação ao desfecho neonatal, a média do Apgar no $1^{\circ}$ minuto de vida foi de $8,2( \pm 1,0)$, e no $5^{\circ}$ minuto $9,1( \pm 0,6)$, condizentes ao encontrado em outros estudos onde os índices estiveram acima do ponto de corte proposto pela literatura ${ }^{(9)}$. Ainda, o peso do Recém Nascido (RN) também obteve média satisfatória de $3.085 \mathrm{Kg}( \pm 458,6)$, bem como a média da idade gestacional ao nascimento de 38,8 semanas $( \pm 1,7)$.

A prematuridade contrapôs aos achados da literatura pertinente em que relata a associação desses fatores com a $\mathrm{SHG}^{(18)}$. Embora a SHG funcione como um fator de estresse para o $\mathrm{RN}$ os achados relacionados à vitalidade fetal extrauterina foram satisfatórios. 
Ainda que os achados deste estudo não mostraram associação com a via de parto, a cesariana foi a mais prevalente $(65,6 \%)$. Seja qual for a forma clínica da SHG a via de parto recomendada pelas diretrizes nacionais e internacionais é o parto vaginal ${ }^{(19)}$. Vale ressaltar que as gestantes neste estudo eram passíveis da realização do parto vaginal.

Por fim, o número de consultas no pré-natal esteve relacionado à ocorrência da SHG, com significância estatística para a PE. Isso reforça a necessidade de monitoramento frequente das gestantes de risco, com o aumento do número de consultas.

Constata-se que a assistência pré-natal alcançou considerável cobertura, com o início precoce no primeiro trimestre e a realização de mais consultas do que o mínimo recomendado. No entanto, foi possível identificar fragilidades no acompanhamento dessas gestantes uma vez que parte das informações clínicas e laboratoriais necessárias para o monitoramento das SHG não foi registrada.

A ausência de preenchimento dos campos do prontuário por si só é um fator preocupante, pois expressa um dos pontos da qualidade na assistência prestada à gestante, já que esse instrumento de registro tem o papel de permitir o fluxo de informações entre os serviços de saúde e o acompanhamento da evolução da gravidez, do parto e do puerpério ${ }^{(2)}$.

\section{CONCLUSÃO}

No universo pesquisado foi possível identificar a complexidade das SHG e observar que alguns fatores estão relacionados com a ocorrência das suas formas clínicas ao longo dos trimestres gestacionais. As características definidoras de cada diagnóstico foram reveladas pelo conjunto de evidências manifestadas por meio da elevação da pressão arterial e presença de proteinúria.

Por outro lado, os fatores relacionados foram: a idade materna, ganho de peso, IMC, antecedentes pessoais de doenças hipertensivas, número de consultas no pré-natal e uso de medicação antihipertensiva, contribuindo para a etiologia do desfecho.

Em relação às formas clínicas a HAC mostrou associação com a ocorrência no primeiro trimestre, seguida da HG no segundo trimestre e PE no terceiro trimestre, está associada à presença de proteinúria, ganho de peso e obesidade. No entanto, o nível socioeconômico, escolaridade, raça/cor, situação ocupacional, paridade, via de parto e prematuridade não mostraram associação significativa.

A identificação das pacientes de risco para o desenvolvimento das SHG pode subsidiar ações para prevenir ou retardar a apresentação clínica das formas mais graves, corroborando com a hipótese inicial deste estudo. Essas observações ainda permanecem como os principais fatores preditores dos distúrbios hipertensivos na gestação.

A captação precoce das gestantes no pré-natal possibilitou a identificação antecipada da gestação de risco, bem como o encaminhamento oportuno para o serviço de referência.

No entanto, a ausência de informações nos prontuários resultou no impedimento de análises de associação de maior impacto sobre a causalidade; como risco relativo por exemplo, configurando uma limitação deste estudo. Também não foi possível o acompanhamento dessas mulheres no puerpério, uma vez que as SHG podem aparecer nesse período.

Apesar da assistência pré-natal estar no escopo das ações praticadas pelos enfermeiros nos serviços de saúde, até o momento, permanecem questões que devem ser discutidas quanto à qualidade da atenção prestada. Tal contexto, induz à reflexão sobre a prática clínica e ao questionamento sobre o que pode ser feito para minimizar os índices da SHG na comunidade de gestantes da região estudada.

Mesmo trabalhando com uma doença complexa e desafiadora os resultados deste estudo oportunizaram conhecer as características definidoras da SHG e a ocorrência da evolução de suas formas clínicas ao longo dos trimestres gestacionais. 


\section{REFERÊNCIAS}

1. World Health Organization (WHO). Recommendations for prevention and treatment of pre-eclampsia and eclampsia. [Internet]. Genebra: WHO; 2011 [acesso em 15 jan 2018]. Disponível: http://apps.who.int/iris/bitstre am/10665/44703/1/9789241548335_eng.pdf.

2. World Health Organization (WHO). Trends in maternal mortality: 1900 to 2015: estimates by WHO, UNICEF, UNFPA, World Bank Group and the United Nations Population Division. [Internet] Genebra: WHO; 2015 [acesso em 15 jan 2018]. Disponível: http://www.afro.who.int/sites/default/files/2017-05/trends-in-maternal-mortality-1990-to-2015.pdf.

3. Ministério da Saúde (BR). Secretaria de Vigilância em Saúde. Departamento de Vigilância de Doenças e Agravos não Transmissíveis e Promoção da Saúde. Saúde Brasil 2014: uma análise da situação de saúde e das causas externas. [Internet] Brasília: Ministério da Saúde; 2015 [acesso em 17 jan 2018]. Disponível: http://bvsms.saude.gov.br/bvs/publicacoes/saude_brasil_2014_analise_situacao.pdf.

4. National Institutes Of Health (NIH). Working group report on high blood pressure in pregnancy. National High Blood Pressure Education Program. [Internet] Bethesda: NIH; 2000 [acesso em 17 nov 2016] Disponível: https://www.nhlbi. nih.gov/files/docs/guidelines/hbp_preg_archive.pdf.

5. Hutcheon JA, Lisonkova S, Joseph KS. Epidemiology of pre-eclampsia and the other hypertensive disorders of pregnancy. Best Practtie \& Research Clinical Obstetrics and Gynecology. [Internet] 2011;25(4) [acesso em 17 nov 2016]. Disponível: https://doi.org/10.1016/j.bpobgyn.2011.01.006.

6. de Moura MDR, Margotto PR, Rugolo LMSS. Alterações do fluxo sanguíneo em artéria umbilical na síndrome hipertensiva gestacional e suas implicações nos resultados neonatais. Rev. Bras. Ginecol. Obstet. [Internet] 2013;35(2) [acesso em 8 nov 2016]. Disponível: http://dx.doi.org/10.1590/S0100-72032013000200006.

7. Myatt L, Redman CW, Staff AC, Hansson S, Wilson ML, Laivuori H, et al. Strategy for Standardization of Preeclampsia Research Study Design. Hypertension. [Internet] 2014;63 [acesso em 14 dez 2017]. Disponível: https://doi.org/10.1161/ HYPERTENSIONAHA.113.02664.

8. Dalmáz CA, dos Santos KG, Botton MR, Roisenberg I. Risk factors for hypertensive disorders of pregnancy in Southern Brazil. Rev. Assoc. Med. Bras. [Internet] 2011;57(6) [acesso em 9 nov 2016]. Disponível: http://dx.doi.org/10.1590/ S0104-42302011000600018.

9. Chapecó (SC). Secretaria da Saúde (SESAU). Plano Municipal de Saúde de Chapecó 2014-2017. [Internet] Chapecó: SESAU; 2014 [acesso em 10 nov 2016]. Disponível: https://secsaude.chapeco.sc.gov.br/uploads/o-planejamento/2/ plano-de-saude-ii-ed-13052015.pdf.

10. American College Of Obstetricians And Gynecologists (ACOG). Hypertension in Pregnancy. [Internet] Washington: ACOG; 2013 [acesso em 18 nov 2016]. Disponível: https://www.acog.org/ /media/Task\%20Force\%20and\%20 Work\%20Group\%20Reports/public/HypertensioninPregnancy.pdf.

11. Oliveira ACM, Santos AA, Bezerra AR, Barros AMR, Tavares MCM. Fatores Maternos e Resultados Perinatais Adversos em Portadoras de Pré-eclâmpsia em Maceió, Alagoas. Arq. Bras. Cardiol. [Internet] 2016;106(2) [acesso em 20 nov 2016]. Disponível: http://www.scielo.br/scielo.php?script=sci_arttext\&pid=S0066-782X2016000200113\&lng= pt\&nrm=iso\&tlng=pt.

12. Romero-Arauz JF, Ortiz-Díaz CB, Leaños-Miranda A, Martínez-Rodrígues OA. Evolución de hypertension gestacional a pré-eclâmpsia. Ginecol. Obstet. Mex. [Internet] 2014;82(4) [acesso em 21 nov 2016]. Disponível: http:// www.medigraphic.com/pdfs/ginobsmex/gom-2014/gom144c.pdf

13. Moodley J, Onyangunga OA, Maharaj NR. Hypertensive disorders in primigravid black South African women: a one-year descriptive analysis. HypertensPregnancy. [Internet] 2016;35(4) [acesso em 22 nov 2016]. Disponível: https:// doi.org/10.1080/10641955.2016.1193190. 
14. Linhares JJ, Macêdo NMQ, de Arruda GM, Vasconcelos JLM, Saraiva TV, Ribeiro AF. Fatores associados à via de parto em mulheres com pré-eclâmpsia. Rev. Bras. Ginecol. Obstet. [Internet] 2014;36(6) [acesso em 25 nov 2016]. Disponível: http://dx.doi.org/10.1590/S0100-720320140004812.

15. Gravena AAF, de Paula MG, Marcon SS, de Carvalho MDB, Pelloso SM. Idade materna e fatores associados a resultados perinatais. Acta paul. enferm. [Internet] 2013;26(2) [acesso em 22 nov de 2016]. Disponível: http://dx.doi. org/10.1590/S0103-21002013000200005.

16. Nama V, Antonios TF, Onwude J, Manyonda IT. Mid-trimester blood pressure drop in normal pregnancy: myth or reality? J. Hypertens. [Internet] 2011;29(4) [acesso em 26 nov 2016]. Disponível: https://www.ncbi.nlm.nih.gov/ pubmed/21178781.

17. Vaiman D, Miralles F. An Integrative Analysis of Pre-eclampsia Based on the Construction of an Extended Composite Network Featuring Protein-Protein Physical Interactions and Transcriptional Relation ships. Plos One. [Internet] 2016;11(11) [acesso em 26 nov 2016]. Disponível: https://doi.org/10.1371/journal.pone.0165849.

18. Venkatesh KK, Cantonwine DE, Zera C, Arjona M, Smith NA, Robinson JN, et al. Is There an Association between Body Mass Index and Cervical Length? Implications for Obesity and Cervical Length Management in Pregnancy. Am. J. Perinatol. [Internet] 2017;34(6) [acesso em 14 fev 2017]. Disponível:http://dx.doi.org/10.1055/s-0036-159424.2

19. Jacob L, Taskan S, Macharey G, Sechet I, Ziller V, Kostev K. Impact of caesarean section on mode of delivery, pregnancy-induced and pregnancy-associated disorders, and complications in the subsequent pregnancy in Germany. GMS German Medical Science. [Internet] 2016;14 [acesso em 27 nov 2016]. Disponível: https://dx.doi. org/10.3205\%2F000233.

20. Goudard MJF, Simões VMF, Batista RFL, Queiroz RCS, Brito e Alves MTSS, Coimbra LC, et al. Inadequação do conteúdo da assistência pré-natal e fatores associados em uma coorte no nordeste brasileiro. Ciência \&Saúde Coletiva. [Internet] 2016;21(4) [acesso em 27 nov 2016]. Disponível: http://dx.doi.org/10.1590/1413-81232015214.12512015. 